\title{
Qualidade de vida em pessoas idosas submetidas ao tratamento de neoplasias
}

\author{
Quality of life in elderly people submitted to the treatment of neoplasms \\ Calidad de vida en personas mayores sometidas altratamiento de neoplasias
}

Recebido: 15/05/2021 | Revisado: 23/05/2021 | Aceito: 25/05/2021 | Publicado: 09/06/2021

\author{
Carlos Rafael Sousa Novais \\ ORCID: https://orcid.org/0000-0002-3944-8772 \\ Faculdade Independente do Nordeste, Brasil \\ E-mail: rafaelnovais.c@gmail.com \\ Luciana Araújo dos Reis \\ ORCID: https://orcid.org/0000-0002-0867-8057 \\ Universidade Estadual do Sudoeste da Bahia, Brasil \\ E-mail: lucianauesb@yahoo.com.br \\ Wagner Couto Assis \\ ORCID: https://orcid.org/0000-0001-7802-2443 \\ Universidade Estadual do Sudoeste da Bahia, Brasil \\ E-mail: wagnerassis2010@hotmail.com \\ Lilian Almeida Nascimento Rabelo \\ ORCID: https://orcid.org/0000-0001-7457-6510 \\ Faculdade de Tecnologia e Ciências, Brasil \\ E-mail: lynascimento@ hotmail.com \\ Frank Evilácio de Oliveira Guimarães \\ ORCID: https://orcid.org/0000-0003-4739-3676 \\ Universidade Católica do Salvador, Brasil \\ E-mail: frankevilacio@hotmail.com \\ Isnara Teixeira de Brito \\ ORCID: https://orcid.org/0000-0001-7916-2085 \\ Universidade Estadual do Sudoeste da Bahia, Brasil \\ E-mail: isnara.britto@hotmail.com \\ Luana Araújo dos Reis \\ ORCID: https://orcid.org/0000-0002-9263-083X \\ Faculdade Independente do Nordeste, Brasil \\ E-mail: luareis1@hotmail.com
}

\begin{abstract}
Resumo
Objetivos: Descrever os impactos do tratamento de neoplasias na qualidade de vida dos idosos, a partir de uma revisão da literatura. Metodologia: Trata-se de um estudo descritivo, de abordagem qualitativa, cujo método utilizado foi à revisão não sistemática de literatura. A revisão foi embasada em referenciais teóricos e artigos científicos publicados no período de 2010 a 2020, disponíveis nas bases de dados virtuais: Scielo, LILACS e Bireme. Os critérios utilizados para a seleção dos artigos foram: artigos escritos no idioma português e com texto completo disponível para acesso gratuito. Resultados: A análise da literatura foi disposta em quatro subtemas: "Envelhecimento e Saúde"; "Qualidade de Vida da Pessoa Idosa"; "Neoplasias e seus Impactos na Saúde da Pessoa Idosa" e "Religiosidade, Espiritualidade e Crenças Pessoais". Considerações Finais: Ao analisar os tópicos abordados é possível verificar que as mudanças na saúde do idoso interfere diretamente na qualidade de vida, principalmente quando submetido ao tratamento oncológico, quando o estado de saúde é ainda mais afetado.
\end{abstract}

Palavras-chave: Qualidade de vida; Pessoa idosa; Neoplasia; Saúde da pessoa idosa.

\begin{abstract}
Objectives: To describe the impacts of cancer treatment on the quality of life of the elderly, based on a literature review. Methodology: This is a descriptive study, with a qualitative approach, whose method used was the nonsystematic literature review. The review was based on theoretical references and scientific articles published from 2010 to 2020, available in the virtual databases: Scielo, LILACS and Bireme. The criteria used for the selection of articles were: articles written in Portuguese and with full text available for free access. Results: The analysis of the literature was arranged in four sub-themes: "Aging and Health"; "Quality of Life for the Elderly"; "Neoplasms and their Impacts on the Health of the Elderly" and "Religiosity, Spirituality and Personal Beliefs". Final Considerations: When analyzing the topics covered, it is possible to verify that changes in the health of the elderly directly interfere in the quality of life, especially when submitted to cancer treatment, when the health status is even more affected.
\end{abstract}

Keywords: Quality of life; Elderly; Neoplasia; Health of the elderly. 


\begin{abstract}
Resumen
Objetivos: Describir los impactos del tratamiento del cáncer en la calidad de vida de las personas mayores, a partir de una revisión de la literatura. Metodología: Se trata de un estudio descriptivo, con enfoque cualitativo, cuyo método utilizado fue la revisión de literatura no sistemática. La revisión se basó en referencias teóricas y artículos científicos publicados de 2010 a 2020, disponibles en las bases de datos virtuales: Scielo, LILACS y Bireme. Los criterios utilizados para la selección de los artículos fueron: artículos redactados en portugués y con texto completo disponible de libre acceso. Resultados: El análisis de la literatura se organizó en cuatro subtemas: "Envejecimiento y salud"; "Calidad de vida de las personas mayores"; "Neoplasias y sus impactos en la salud de las personas mayores" y "Religiosidad, espiritualidad y creencias personales". Consideraciones finales: Al analizar los temas tratados, es posible verificar que los cambios en la salud de los ancianos interfieren directamente en la calidad de vida, especialmente cuando se someten a tratamiento oncológico, cuando el estado de salud se ve aún más afectado.
\end{abstract}

Palabras clave: Calidad de vida; Anciano; Neoplasia; Salud de las personas mayores.

\title{
1. Introdução
}

O envelhecimento populacional é um processo que vem acontecendo rapidamente no Brasil e a tendência é de se acentuar cada vez mais. Sendo também um novo desafio para a saúde pública. Além disso, devido a alterações funcionais próprias dos idosos, eles apresentam como fator de risco maior índice de desenvolver células neoplásicas (Silva; Prá, 2014).

O câncer é uma das mais temidas doenças crônicas não transmissíveis (DCNT), apesar que tenha possibilidade de cura, quando diagnosticado precocemente. Com a idade a incidência dessa doença aumenta consideravelmente. Com o acúmulo dos fatores de risco o idoso apresenta uma menor adesão ao tratamento oncológicos, assim refletindo em maiores efeitos colaterais durante o período de exposição terapêutica interferindo diretamente na qualidade de vida do idoso (Oliveira et al., 2015).

Um estudo brasileiro realizado no Sul do país, por Brustolin e Ferretti (2016), apontou que na maioria dos casos, o câncer ocorre após os 60 anos. Essa maior incidência no idoso se deve ao fato de que cerca de $80 \%$ de todos os cânceres estão relacionados, direta ou indiretamente, ao tempo de exposição a agentes cancerígenos. Menezes (2018), reforça que o câncer pode interferir, de modo negativo, na percepção da qualidade de vida, por isso a sua avaliação é considerada um critério importante na área da oncologia.

As transições demográfica e epidemiológica sinalizam para a importância crescente do câncer nas próximas décadas. Enquanto causa de óbito, na maioria dos países, e no Brasil, é suplantado apenas pelas doenças cardiovasculares. Estima-se que em 2025 a carga do câncer aumentará em 50\% devido ao envelhecimento populacional e ao aumento dos fatores de risco no estilo de vida (Menezes et al., 2018), o que impacta na qualidade de vida.

Segundo Paula e Sawada (2015), o termo Qualidade de Vida Relacionada à Saúde (QVRS) é evidenciado na literatura como sinônimo de Estado de Saúde Percebido e tem como objetivo principal verificar o quanto a doença ou estado crônico, além de seus sintomas, passam a interferir na vida diária de um indivíduo, portanto, Qualidade de Vida Relacionada à Saúde pode ser definido como Qualidade de Vida dentro do contexto de saúde.

A qualidade de vida é considerada como a percepção subjetiva dos aspectos importantes na vida de uma pessoa, fatores que interferem o padrão de vida. Com o aumento da detecção precoce do câncer, a avaliação da qualidade de vida vem contribuindo significativamente para o seu atendimento, podendo ser medida objetivamente para avaliar os efeitos de diferentes tratamentos sobre as funções essenciais (Ferreira; Meireles; Ferreira, 2018).

Tem como objetivo descrever os impactos do tratamento de neoplasias na qualidade de vida dos idosos, a partir de uma revisão da literatura. 


\section{Metodologia}

Para alcançar o objetivo proposto, optou-se pela pesquisa descritiva, de abordagem qualitativa, cujo método utilizado foi à revisão não sistemática de literatura. A pesquisa descritiva tem como principal objetivo descrever características de determinada população ou fenômeno ou estabelecimento de relações entre variáveis. Uma das suas características mais importantes está na utilização de padrões de coleta de dados (Marconi; Lakatos, 2007).

O método qualitativo não aplica instrumentos estatísticos para análise de um problema, uma vez que seu objetivo não é medir nem numerar os eventos estudados. A pesquisa qualitativa consiste em "um conjunto de práticas interpretativas que faz o mundo visível". Esse tipo de pesquisa busca a obtenção de dados descritivos de pessoas, lugares e processos interativos que acontece através do contato direto do pesquisador com aquilo que está sendo estudado, sendo que a compreensão dos fenômenos se dá segundo a perspectiva dos sujeitos participantes (Paschoarelli; Medola; Bonfim, 2018).

A construção da pesquisa foi embasada em referenciais teóricos e artigos científicos publicados no período de 2010 a 2020, disponíveis nas bases de dados virtuais: Scielo, LILACS e Bireme. Os artigos foram levantados através da consulta com os descritores padronizados pelo Descritores em Ciências da Saúde (Decs): Qualidade de vida; Pessoa idosa; Neoplasia; Saúde da pessoa idosa. Os critérios utilizados para a seleção dos artigos foram: artigos no idioma português e disponíveis na íntegra.

As impressões e considerações da análise da literatura serão dispostas em quatro subtemas "Envelhecimento e Saúde", "Qualidade de vida da pessoa idosa", "Neoplasias e seus Impactos na Saúde da Pessoa Idosa" e "Religiosidade, Espiritualidade e Crenças pessoais". Dessa forma, esse estudo objetiva descrever os impactos dos fatores extrínsecos no envelhecimento precoce, a partir de uma revisão da literatura.

\section{Resultados e Discussão}

\section{Envelhecimento e Saúde}

O envelhecimento, processo dinâmico, progressivo e irreversível, pelo qual perpassa o mundo atualmente, traz consigo importantes modificações fisiológicas nos diferentes órgãos e sistemas corporais, as quais podem refletir diretamente na saúde do ser humano. Dentre estas destacamos as doenças crônicas não transmissíveis (DCNT), sendo o câncer uma das mais incidentes neste segmento populacional. O envelhecimento e a oncologia estão intimamente relacionados, pois diversas funções celulares são alteradas progressivamente com a idade, aumentando a suscetibilidade à transformação maligna. Devido a um aumento no número de casos nesta faixa etária, pode-se inferir que, à medida que a população envelhece as estimativas para os novos casos se confirmam (Januário et al., 2018).

Em tempos atuais a população mundial cresceu de forma a se tornar um fenômeno de amplitude global, a Organização Mundial de Saúde (OMS) prevê que em 2025 existirão 1,2 biliões de pessoas com mais de 60 anos, sendo que os muito idosos (com 80 ou mais anos) constituem o grupo etário de maior crescimento (OMS, 2001). Ainda em concordância com mesma fonte de informação citada a maior parte dessas pessoas (aproximadamente 75\%) vive nos países desenvolvidos.

Estima-se que 26 milhões de novos casos de câncer serão diagnosticados no mundo até 2030 e que mais de $50 \%$ desses casos ocorrerão em idosos, que é também o grupo de maior taxa de mortalidade por essa doença (Ferreira et al., 2015).

\section{Qualidade de Vida da Pessoa Idosa}

De acordo com a definição do Grupo de Qualidade de Vida criado pela OMS, qualidade de vida nada mais é que a percepção do indivíduo de sua posição na vida, no contexto da cultura e sistema de valores nos quais ele vive e em relação aos seus objetivos, expectativas, padrões e preocupações (Amadeu; Justi, 2017). 
Uma ferramenta de essencial compreensão é a avaliação da qualidade de vida de pacientes em tratamento oncológico possibilita melhor compreensão da intensidade e quantidade dos sintomas do paciente e da significativa relevância que isso tem na sua saúde, permitindo também melhorar o planejamento da estratégia terapêutica. (Ferreira et al., 2015).

Ainda segundo os mesmos autores, a manutenção da qualidade de vida tem sido o objetivo primordial do tratamento para parte dos pacientes idosos com câncer, sobrepondo-se inclusive sobre o aumento de sobrevida, visto que essa população tem riscos mais elevados de morte além de câncer. Contudo a toxicidade do tratamento abala de forma mais efetiva o paciente idoso do que um jovem adulto, por exemplo, colocando em evidente discussão a melhor opção terapêutica.

Segundo Instituto Nacional de Câncer (2011), nas últimas décadas tem sido observado um aumento significativo na magnitude das neoplasias malignas. Por esse motivo o perfil das enfermidades tem se modificado bastante. Tornou-se necessário ampliar os cuidados de saúde oferecidos às pessoas acometidas de enfermidades crônicas avançadas. Reforçando uma resposta ativa a todos os problemas gerados pela progressão das doenças avançadas crônicas e que não tem cura.

Um tema complexo, a qualidade de vida pode variar significativamente entre indivíduos e culturas. A qualidade de vida é alcançada através de ações intrínsecas, autodeterminadas como a busca de bons motivos para conviver com o câncer em tratamento; concretizar ações de manutenção preventiva em saúde de forma a manter a realização de atividades de seu cotidiano, que não são necessariamente atividades básicas de vida diária; e, manter a saúde mental através da busca ativa pela vontade de viver com paz interior, com alegria e de bem com a vida (Silva; Hansel; Da Silva, 2016).

\section{Neoplasias e seus Impactos na Saúde da Pessoa Idosa}

Entre as doenças crônico degenerativas, o câncer ocupa lugar de destaque nesse contexto. Com mais de 11 milhões de casos novos e sete milhões de mortes, por ano, no mundo. Portanto, trata-se de uma doença de alta prevalência global. Verifica-se também um maior índice de mortalidade acima dos 30 de idade, em especial na população idosa. O Ministério da Saúde estimou, em 2002, 128.162 óbitos por câncer, com 81.152 (63\%) destes óbitos ocorrendo na população acima de sessenta anos (Floriani; Schramm, 2008).

Considerando a distribuição dos óbitos por tipos de câncer segundo o sexo nos idosos, para o período de 1996-2016, os principais entre os homens corresponderam à neoplasia maligna da próstata (18\%), seguido por neoplasia maligna de traqueia, brônquios e pulmões (12\%). Nas mulheres, as neoplasias malignas da mama representaram $12 \%$ e de traqueia, brônquios e pulmões corresponderam a 11\%. Estimativas da incidência de câncer no Brasil, realizada pelo Instituto Nacional do Câncer (INCA), apontam para cada ano do triênio 2020-2022, um total de 625 mil casos novos, com maior incidência para o câncer de pele não melanoma (177 mil), seguido pelos cânceres de mama e próstata (66 mil cada) (Francisco et al., 2020).

Os sinais e sintomas pouco controláveis como dor, náuseas, vômitos, anorexia, fadiga, depressão, ansiedade, constipação, entre outros são os que mais afetam os idosos em fases mais avançadas das neoplasias. As manifestações podem estar relacionadas à invasão tumoral, como também aos efeitos adversos do tratamento em alguns tipos de câncer, causando intenso desconforto ao paciente e um impacto circunstancialmente negativo para a qualidade de vida (Freire et al., 2018).

De acordo com a OMS, é importante a participação da família e amigos próximos no processo de aceitação e suporte do paciente. Além do suporte profissional, o envolvimento da família e amigos, quando possível, é sempre positivo. A presença da família oferece segurança e confiabilidade para o paciente em relação aos cuidados que estão sendo tomados para sua patologia e, muitas vezes, também participa.

Dentre as habilidades que o profissional de saúde deve ter uma delas é respeitar a fragilidade dessa condição humana, a dimensionar a fragilidade física e psicológica do paciente e do profissional em relação aos valores pessoais e espirituais. Para além da competência técnica profissional, nada substitui a pessoa humana como fonte e fator de cura para o doente (Júnior; Reis, 2007). 


\section{Religiosidade, Espiritualidade e Crenças pessoais}

A pessoa idosa quando é diagnosticada com o câncer, passa por modificação das percepções, alterações orgânicas, consequência dessa nova condição, modificando o estilo de vida. Além disso, o diagnóstico de câncer carrega consigo estigmas que podem ter um efeito devastador, além de implicações relacionadas à perda da produtividade e das capacidades físicas das pessoas. A religiosidade e a espiritualidade são importantes recursos utilizados no enfrentamento do câncer em todo o mundo, embora sejam pouco explorados nas diferentes culturas (Freitas et al., 2020).

A espiritualidade e religiosidade são consideradas importantes estratégias de enfrentamento interferindo diretamente no bem-estar psicológico, como felicidade, satisfação com a vida, bom desempenho na saúde física e mental, entre outros, para pacientes idosos com alguma neoplasia (Oliveira et al., 2018).

Segundo Menezes (2018), as facetas com maior destaque na avaliação da qualidade de vida são: fé e conexão com o ser ou força espiritual. Observou-se que a fé é considerada como apoio fundamental para superar as situações difíceis, colabora com o bem-estar no dia a dia e na relação com os outros.

Observou-se com os estudos que as crenças proporcionam ao paciente uma mudança no enfrentamento da doença, modificando a forma como o paciente e as pessoas próximas a este, visualizam o problema, gerando alívio da dor, estresse e temor (Bittar; Cassiano; Silva, 2018).

\section{Considerações Finais}

Com base nas discussões levantadas através da literatura analisada é possível perceber que as mudanças na saúde do idoso interferem diretamente na qualidade de vida, principalmente quando submetido ao tratamento oncológico, quando o estado de saúde é ainda mais afetado. Ademais, os resultados demonstraram, ainda, que a pessoa idosa lança mão da religiosidade, espiritualidade e crenças pessoais como estratégias para o enfrentamento dos problemas de saúde, sobretudo em razão dos impactos negativos que o câncer ocasiona na qualidade de vida.

Portanto, a compreensão e discussão sobre esse tema é de extrema relevância, pois conhecer a realidade das alterações e dificuldades na qualidade de vida dos idosos em tratamento oncológico refletirá em um menor impacto dos fatores socioeconômicos, nível de dependência e dados clínicos.

Frente ao exposto, sugere-se a realização de novos estudos que tornem possível a identificação do ponto de vista do paciente em relação a esse contexto, a fim de trazer mais evidencias aos resultados encontrados e, consequentemente, subsidiar a atuação dos profissionais de saúde no contexto da atenção à saúde da pessoa idosa.

\section{Referências}

Amadeu, J., \& Justi, M. (2017). Qualidade de vida de estudantes de graduação e pós-graduação em Odontologia. Arch Health Invest, 6(11), 540-544.

Bittar, C. L., Cassiano, R. V., \& Silva, L. N. (2018). Espiritualidade e religiosidade como estratégia de enfrentamento do câncer de mama: relato de um grupo de pacientes. Psicologia da Saúde, 26 (2).

Brustolin, A., \& Ferretti, F. (2016). Câncer em idosos: a sobrevivência em foco. FisiSenectus, 4(2),1-2.

Ferreira L., Meireles J., \& Ferreira M. (2018). Avaliação do estilo e qualidade de vida em idosos: uma revisão de literatura. Revista Brasileira de Geriatria Gerontologia, 21(5), 639-651.

Ferreira, M. L. L., et al. (2015). Qualidade de vida relacionada à saúde de idosos em tratamento quimioterápico. Revista Brasileira de Geriatria Gerontologia, $18(1), 165-177$

Floriani, C. A., \& Schramm, F. R. (2008). Cuidados paliativos: interfaces, conflitos e necessidades. Ciência \& Saúde Coletiva, 13(2), $2123-2132$.

Francisco, P. M. S. B. et al. (2020). Prevalência de diagnóstico e tipos de câncer em idosos: dados da Pesquisa Nacional de Saúde. Revista Brasileira de Geriatria Gerontologia, 23(2).

Freire, M. E. M. et al. (2018). Qualidade de vida relacionada à saúde de pacientes com câncer em cuidados paliativos. Revista de Enfermagem [online], 27(2). 
Research, Society and Development, v. 10, n. 6, e57610616137, 2021

(CC BY 4.0) | ISSN 2525-3409 | DOI: http://dx.doi.org/10.33448/rsd-v10i6.16137

Freitas, R. A. et al. (2020) Espiritualidade e religiosidade no vivido do sofrimento, culpa e morte da pessoa idosa com câncer. Revista Brasileira de Enfermagem, Epub, July 08.

Instituto Nacional de Câncer (Brasil). (2011). ABC do câncer: abordagens básicas para o controle do câncer / Instituto Nacional de Câncer. IL.ISBN, 97885-7318-188-3.

Januário, I. S. et al. (2018). Repercussão do diagnóstico de câncer em idosos no seio familiar. Revista Cubana de Enfermeira, 34(1), 73-85.

Júnior, L. C. R., \& Reis, P. E. A. M. (2007) Cuidados paliativos no paciente idoso: o papel do fisioterapeuta no contexto multidisciplinar. Fisioterapia em Movimento, 20(2), 127-135.

Marconi, M., \& Lakatos, E.(2007). Metodologia cientifica. Atlas.

Menezes, R. R., et al. (2018). Qualidade de Vida Relacionada à Saúde e Espiritualidade em Pessoas com Câncer. Revista Brasileira de Cancerologia, 64(1), 917.

Oliveira, D. S., et al. (2018). Influência da espiritualidade, religiosidade e crenças pessoais na qualidade de vida de pacientes em quimioterapia. Saúde em temas 18(2), 2447-2131.

Oliveira, M. M. et al. (2015). Estimativa de pessoas com diagnóstico de câncer no Brasil: dados da Pesquisa Nacional de Saúde. Revista Brasileira de Epidemiologia, 18(supp12), 146-157.

Paschoarelli, L., Medola, F., \& Bonfim, G. (2018). Características Qualitativas, Quantitativas e Quali quantitativas de Abordagens Científicas: estudos de caso na subárea do Design Ergonômico. Revista de Design, Tecnologia e Sociedade. 2(1), 65-78.

Paula, J. M., \& Sawada, N. O. (2015). Qualidade de vida relacionada à saúde de pacientes com câncer em tratamento radioterápico. Rev Rene, 16(1), 106-13.

Silva, A., \& Prá, K. R. D. (2014). Envelhecimento populacional no Brasil: elementos para pensar o lugar das famílias na proteção dos idosos. Argumentum, 6(1), 99-115.

Silva, J. A. S., Hansel, G. H., \& Da Silva, J. (2016). Qualidade de vida na perspectiva de idosos com câncer: implicações para enfermagem na atenção básica. Revista de enfermagem UERJ, 24(3), e9621.

Sousa, L., Galante, H., \& Figueiredo D. (2003). Qualidade de vida e bem-estar dos idosos: um estudo exploratório na população portuguesa. Revista de Saúde Pública, 37(3), 364-71. 